\title{
Liquid Crystalline Bolaamphiphiles with Semiperfluorinated Lateral Chains: Competition Between Layer-Like and Honeycomb-Like Organization
}

Xiaohong Cheng, ${ }^{\dagger}$ Malay Kumar Das, ${ }^{\ddagger}$ Ute Baumeister, ${ }^{\ddagger}$ Siegmar Diele, ${ }^{\ddagger}$ and Carsten Tschierske*t

${ }^{\dagger}$ Institute of Organic Chemistry, University Halle, Kurt-Mothes-Str.2, D-06120 Halle,

Germany

${ }^{\ddagger}$ Institute of Physical Chemistry, University Halle, Mühlpforte 1, D-06108 Halle, Germany

\section{Supporting Information}

\section{Experimental conditions for $\mathbf{X}$-ray scattering}

Powder X-ray investigations were carried out with a Guinier-Goniometer (Huber). Samples in glass capillaries $(\varnothing 1 \mathrm{~mm})$ in a temperature-controlled heating stage, quartz-monochromatized $\mathrm{CuK}_{\alpha}$ radiation, 30 min exposure time, calibration with the powder pattern of $\mathrm{Pb}\left(\mathrm{NO}_{3}\right)_{2}$, indices according to the two-dimensional lattices as found for samples, aligned at the surface of a glass plate or at the sample-air interface and recorded with a 2D detector (HI-STAR, Siemens, Nimonochromatized $\mathrm{CuK}_{\alpha}$ radiation).

$\theta_{\exp }=$ Bragg angle measured from the Guinier film patterns

$\theta_{\text {calc }}=$ Bragg angle calculated from the lattice parameters for all observed reflections (by Guinier film as well as by 2D detector) according to:

$$
\sin ^{2} \theta_{\text {calc }}=\frac{\lambda^{2}}{4}\left\{\left(\frac{h^{2}}{a^{2}}\right)+\left(\frac{k^{2}}{b^{2}}\right)\right\}
$$




\section{$1.1 \mathrm{Col}_{\mathrm{r} 2} / \mathrm{c} 2 \mathrm{~mm}$ phase of $2 / 7$}

Table S1: $\quad$ Compound 2/7, rectangular columnar phase at $90^{\circ} \mathrm{C}$. Observed reflections: $h k$ with $h+k=2 n$, assumed plane group: $c 2 m m$ Lattice parameters $a=3.00 \mathrm{~nm}, b=8.21 \mathrm{~nm}$

\begin{tabular}{|c|c|c|c|c|}
\hline $\mathrm{h}$ & $\mathrm{k}$ & $\theta_{\exp }\left({ }^{\circ}\right)$ & $\theta_{\text {calc }}\left(^{\circ}\right)$ & $\theta_{\exp }-\theta_{\text {calc }}\left(^{\circ}\right)$ \\
\hline 0 & 2 & 1,08 & 1,08 & 0.00 \\
\hline 1 & 1 & 1,58 & 1,57 & 0.01 \\
\hline 0 & 4 & 2,15 & 2,15 & 0.00 \\
\hline 1 & 3 & 2,19 & 2,19 & 0.00 \\
\hline 2 & 0 & 2,95 & 2,95 & 0.00 \\
\hline 2 & 2 & 3,14 & 3,14 & 0.00 \\
\hline 0 & 6 & 3,25 & 3,23 & 0.02 \\
\hline 3 & 1 & 4,45 & 4,45 & 0.00 \\
\hline
\end{tabular}

\section{2 $\mathrm{Col}_{\mathrm{r} 1} / \mathrm{c2mm}$ phase of $2 / 7$}

Table S2: $\quad$ Compound 2/7, rectangular columnar phase at $115^{\circ} \mathrm{C}$. Observed reflections: $h k$ with $h+k=2 n$, assumed plane group: $c 2 m m$ Lattice parameters $a=2.96 \mathrm{~nm}, b=5.59 \mathrm{~nm}$

\begin{tabular}{|c|c|c|c|c|}
\hline $\mathrm{h}$ & $\mathrm{k}$ & $\theta_{\exp }\left({ }^{\circ}\right)$ & $\theta_{\text {calc }}\left({ }^{\circ}\right)$ & $\theta_{\exp }-\theta_{\text {calc }}\left({ }^{\circ}\right)$ \\
\hline 0 & 2 & 1.58 & 1.58 & 0.00 \\
\hline 1 & 1 & 1.69 & 1.69 & 0.00 \\
\hline 1 & 3 & $2.81^{\mathrm{a}}$ & 2.80 & 0.01 \\
\hline 2 & 0 & $3.00^{\mathrm{a})}$ & 2.99 & 0.01 \\
\hline 0 & 4 & $3.11^{\mathrm{a}}$ & 3.16 & -0.05 \\
\hline 2 & 2 & $3.38^{\mathrm{a})}$ & 3.38 & 0.00 \\
\hline 1 & 5 & $\left.4.21^{\mathrm{a}}\right)$ & 4.23 & -0.02 \\
\hline 2 & 4 & $4.31^{\mathrm{a})}$ & 4.35 & -0.04 \\
\hline 0 & 6 & $4.67^{\mathrm{a})}$ & 4.75 & -0.08 \\
\hline
\end{tabular}




\section{$1.3 \mathrm{Col}_{\mathrm{r} 3} / \mathrm{c} 2 \mathrm{~mm}$ phase of $2 / 4$}

Table S3: $\quad$ Compound 2/4, rectangular columnar phase at $110^{\circ} \mathrm{C}$. Observed reflections: $h k$ with $h+k=2 n$, assumed plane group: $c 2 m m$ Lattice parameters $a=3.13 \mathrm{~nm}, b=4.62 \mathrm{~nm}$

\begin{tabular}{|c|c|c|c|c|}
\hline $\mathrm{h}$ & $\mathrm{k}$ & $\theta_{\exp }\left({ }^{\circ}\right)$ & $\theta_{\text {calc }}\left({ }^{\circ}\right)$ & $\theta_{\exp }-\theta_{\text {calc }}\left({ }^{\circ}\right)$ \\
\hline 1 & 1 & 1.70 & 1.70 & 0.00 \\
\hline 0 & 2 & 1.91 & 1.91 & 0.00 \\
\hline 2 & 0 & $\left.2.83^{\mathrm{a}}\right)$ & 2.82 & 0.01 \\
\hline 1 & 3 & $3.19^{\mathrm{a})}$ & 3.20 & -0.01 \\
\hline 2 & 2 & $3.40^{\mathrm{a})}$ & 3.41 & -0.01 \\
\hline 0 & 4 & $3.82^{\mathrm{a})}$ & 3.83 & -0.01 \\
\hline 2 & 4 & $4.75^{\mathrm{a})}$ & 4.76 & -0.01 \\
\hline 4 & 0 & $5.65^{\mathrm{a})}$ & 5.65 & 0.00 \\
\hline 0 & 6 & $5.73^{\mathrm{a})}$ & 5.75 & -0.02 \\
\hline 4 & 2 & $5.97^{\mathrm{a})}$ & 5.97 & 0.00 \\
\hline
\end{tabular}

\section{$1.3 \mathrm{Col}_{\mathrm{r}} / \mathrm{p} 2 \mathrm{~mm}$ phase of $2 / 3$}

Table S4: $\quad$ Compound $2 / 3$, rectangular columnar phase at $92{ }^{\circ} \mathrm{C}$.

Assumed plane group: $p 2 \mathrm{~mm}$

Lattice parameters $a=1.57 \mathrm{~nm}, b=2.16 \mathrm{~nm}$

\begin{tabular}{|c|c|c|c|c|}
\hline $\mathrm{h}$ & $\mathrm{k}$ & $\theta_{\exp }\left({ }^{\circ}\right)^{a}$ & $\theta_{\text {calc }}\left({ }^{\circ}\right)$ & $\theta_{\exp }-\theta_{\text {calc }}\left({ }^{\circ}\right)$ \\
\hline 0 & 1 & $2.05^{\mathrm{a})}$ & 2.05 & 0.00 \\
\hline 1 & 0 & $2.81^{\mathrm{a})}$ & 2.81 & 0.00 \\
\hline 1 & 1 & $3.43^{\mathrm{a})}$ & 3.48 & -0.05 \\
\hline
\end{tabular}

${ }^{a}$ Values from 2D patterns. 


\section{Textural changes at the phase transitions of compound $2 / 7$}
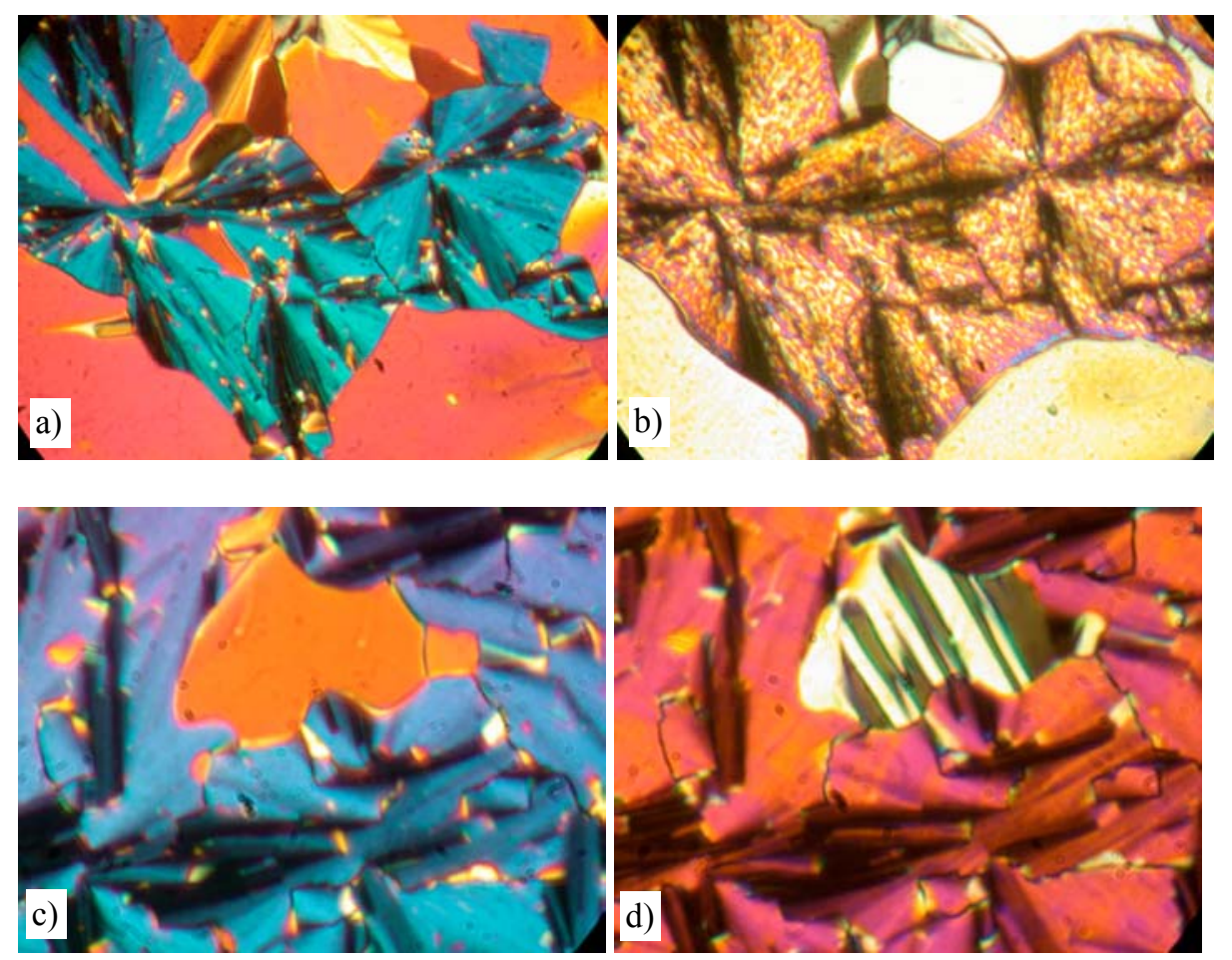

Figure S1. Photomicrographs (crossed polarizers) of the mesophases of compound 2/7: (a) $\mathrm{Col}_{\mathrm{r}}$ phase $(\mathrm{c} 2 \mathrm{~mm})$ at $130^{\circ} \mathrm{C}$. (b) transition from the $\mathrm{Col}_{\mathrm{r}} / \mathrm{c} 2 \mathrm{~mm}$ phase to the $\mathrm{Lam}_{\mathrm{Sm}}$ phase at $131^{\circ} \mathrm{C}$ upon heating; (c) $\mathrm{Col}_{\mathrm{r} 1}$ phase $(\mathrm{c} 2 \mathrm{~mm})$ at $110^{\circ} \mathrm{C}$. (f) $\mathrm{Col}_{\mathrm{r} 2}$ phase $(\mathrm{c} 2 \mathrm{~mm})$ at $95^{\circ} \mathrm{C}$.

\section{Synthesis of compounds $2 / n$}

Unless otherwise noted, all starting materials were purchased from commercial sources and were used as obtained. Preparative thin layer chromatography was performed with a Chromatotron (Harrison-Research) using silica gel $60 \mathrm{PF}_{254}$ (Merck). Column chromatography was performed with silica gel 60 (0.063 - 0.2, Merck). Confirmation of the structures and purity of intermediates and products was obtained by NMR spectroscopy (VARIAN Unity 500, VARIAN Gemini 200 and VARIAN Gemini 2000 spectrometers, all spectra were recorded at $27^{\circ} \mathrm{C}$ ). Microanalyses were performed using a LECO CHNS-932 elemental analyzer. The purity of all compounds was checked by thin layer chromatography (Merck, silicagel $60 \mathrm{~F}_{254}$ ). Hexane/EtOAc mixtures and $\mathrm{CHCl}_{3} / \mathrm{MeOH}$ mixtures were used as eluent and the spots were detected by UV radiation.

Transition temperatures were measured by means of a Nikon Optiphot polarizing microscope with Linkam LTS 350 hotstage and control unit and confirmed using differential scanning calorimetry (Perkin Elmer DSC-7). 
For 2/7 as representative example the synthesis is described in full detail. All other compounds $2 / n$ and $3 / n$ were synthesized in an analogous manner. For these compounds the analytical data of the final products are provided.

\subsection{Synthesis of compound $2 / 7$}

\subsubsection{2-(1H,1H,2H,2H,3H,3H-Perfluorodecyl)phenol}

2-Allylphenol (4.5 g, $33.6 \mathrm{mmol})$, 1-iodoperfluoroheptane (25 g, $50.4 \mathrm{mmol})$ and dry hexane $(30 \mathrm{~mL})$ were put into a dry three necked flask, the flask was placed in an ultra sonic bath under an argon atmosphere for $5 \mathrm{~min}$. Then the mixture was cooled to $-78{ }^{\circ} \mathrm{C}$ and the flask was evacuated and refilled with argon, and then warmed up to room temperature. This procedure was repeated for three times. The mixture was cooled to $0{ }^{\circ} \mathrm{C},\left(\mathrm{Ph}_{3} \mathrm{P}\right)_{4} \mathrm{Pd}(1.4 \mathrm{~g})$ was added under inert conditions and the heterogeneous orange reaction mixture was allowed to reach room temperature while stirring. Stirring was continued at $25^{\circ} \mathrm{C}$ until the reaction was completed $(36 \mathrm{~h})$. The mixture was filtered through silica gel and the residue was washed thoroughly with $\mathrm{Et}_{2} \mathrm{O}(100 \mathrm{~mL})$. The solvent was removed in vacuo, and the residue was dissolved in dry $\mathrm{Et}_{2} \mathrm{O}$. This solution was added dropwise to a vigorously stirred slurry of $\mathrm{LiAlH}_{4}(1.5 \mathrm{~g})$ in dry $\mathrm{Et}_{2} \mathrm{O}(80 \mathrm{~mL})$ at such a rate to maintain the solution at reflux. The mixture was refluxed for additional $2 \mathrm{~h}$ and then cooled to $5^{\circ} \mathrm{C}$ (ice bath). Afterwards, water $(5 \mathrm{~mL})$ was added dropwise until all the unreacted $\mathrm{LiAlH}_{4}$ was decomposed (CAUTION! strongly exothermic reaction). Then $30 \%$ aqueous $\mathrm{H}_{2} \mathrm{SO}_{4}(20 \mathrm{~mL})$ was carefully added to dissolve all precipitated inorganic solids. The organic layer was separated and the aqueous layer was extracted with $\mathrm{Et}_{2} \mathrm{O}(3 \times 100 \mathrm{~mL})$, the organic layers were combined and washed with $10 \%$ aqueous $\mathrm{Na}_{2} \mathrm{~S}_{2} \mathrm{O}_{3}$ until the aqueous layer remained colorless. After washing with $\mathrm{H}_{2} \mathrm{O}(2 \times 100 \mathrm{~mL})$ and brine $(2 \times 100 \mathrm{~mL})$ the solution was dried over $\mathrm{Na}_{2} \mathrm{SO}_{4}$, the solvent was removed in vacuo and the oily residue was distilled in vacuo. Yield: $11.9 \mathrm{~g}(70.3 \%)$; colorless solid; mp: $56{ }^{\circ} \mathrm{C}$, bp: $115^{\circ} \mathrm{C}$ at $0.2 \mathrm{mbar} ;{ }^{1} \mathrm{H}-\mathrm{NMR}\left(200 \mathrm{MHz} ; \mathrm{CDCl}_{3} ; \mathrm{J} / \mathrm{Hz}\right): \delta=7.1(\mathrm{~m}, 2 \mathrm{H}$, Ar-H), 6.9 (m, $1 \mathrm{H}, \mathrm{Ar}-\mathrm{H}), 6.7(\mathrm{~m}, 1 \mathrm{H}, \mathrm{Ar}-\mathrm{H}), 4.67(\mathrm{~s}, 1 \mathrm{H}, \mathrm{OH}), 2.71\left(\mathrm{t},{ }^{3} J(\mathrm{H}, \mathrm{H})\right.$ 7.3, $2 \mathrm{H}$, Ar- $\left.\mathrm{CH}_{2}\right), 1.8-2.3\left(\mathrm{~m}, 4 \mathrm{H}, \mathrm{CH}_{2} \mathrm{CH}_{2} \mathrm{CF}_{2}\right)$.

\subsubsection{4-Bromo-2-(1H,1H,2H,2H,3H,3H-perfluorodecyl)phenol}

A mixture of $2-(1 \mathrm{H}, 1 \mathrm{H}, 2 \mathrm{H}, 2 \mathrm{H}, 3 \mathrm{H}, 3 \mathrm{H}$-perfluorodecyl $)$ phenol $(11.9 \mathrm{~g}, 23.6 \mathrm{mmol})$, acetic acid $(70 \mathrm{~mL})$ and $33 \% \mathrm{HBr}$ in acetic acid $(30 \mathrm{~mL})$ was cooled in an ice bath to $-5{ }^{\circ} \mathrm{C}$. Then, DMSO $(30 \mathrm{~mL})$ was added dropwise. The mixture was stirred at $5-10{ }^{\circ} \mathrm{C}$ for $30 \mathrm{~min}$, then saturated aqueous $\mathrm{NaHCO}_{3}$ solution $(100 \mathrm{~mL})$ was added to quench the reaction. The mixture was extracted with $\mathrm{Et}_{2} \mathrm{O}(3 \times 100 \mathrm{~mL})$. The combined organic layers were washed with saturated aqueous $\mathrm{NaHCO}_{3}$ solution until $\mathrm{pH}=6-7$. Then the solution was washed with $\mathrm{H}_{2} \mathrm{O}$ $(3 \times 75 \mathrm{~mL})$, brine $(3 \times 75 \mathrm{~mL})$, dried over $\mathrm{Na}_{2} \mathrm{SO}_{4}$ and the solvent was evaporated. Recrystallization from hexane, yielded the pure product. Yield: $7.5 \mathrm{~g}$ (54.9\%); colorless solid; mp: $49{ }^{\circ} \mathrm{C} ;{ }^{1} \mathrm{H}-\mathrm{NMR}\left(200 \mathrm{MHz} ; \mathrm{CDCl}_{3} ; \mathrm{J} / \mathrm{Hz}\right.$ ): $\delta=7.1-7.3(\mathrm{~m}, 3 \mathrm{H}, \mathrm{Ar}-\mathrm{H}), 5.03$ (s br, 1 $\mathrm{H}, \mathrm{OH}), 2.66\left(\mathrm{t},{ }^{3} J(\mathrm{H}, \mathrm{H}) 7.3,2 \mathrm{H}, \mathrm{Ar}-\mathrm{CH}_{2}\right), 1.8-2.3\left(\mathrm{~m}, 4 \mathrm{H}, \mathrm{CH}_{2} \mathrm{CH}_{2} \mathrm{CF}_{2}\right)$.

\subsubsection{1-Allyloxy-4-bromo-2-(1H,1H,2H,2H,3H,3H-perfluorodecyl)benzene}

Allylbromide $(2.3 \mathrm{~g}, 19.0 \mathrm{mmol})$ was added to a mixture of 4-bromo-2$\left(1 \mathrm{H}, 1 \mathrm{H}, 2 \mathrm{H}, 2 \mathrm{H}, 3 \mathrm{H}, 3 \mathrm{H}\right.$-perfluorodecyl)phenol $(7.5 \mathrm{~g}, 12.9 \mathrm{mmol})$ and $\mathrm{K}_{2} \mathrm{CO}_{3}(4.0 \mathrm{~g}, 29.0$ $\mathrm{mmol})$ in dry $\mathrm{MeCN}(50 \mathrm{~mL})$, while stirring under an argon atmosphere. The mixture was refluxed for $2 \mathrm{~h}$ and then the solvent was distilled off. $\mathrm{H}_{2} \mathrm{O}(100 \mathrm{~mL})$ and $\mathrm{Et}_{2} \mathrm{O}(100 \mathrm{~mL})$ were added to the residue. The organic layer was separated, and the aqueous layer was extracted with $\mathrm{Et}_{2} \mathrm{O}(2 \times 50 \mathrm{~mL})$ and the combined extracts were washed with $\mathrm{H}_{2} \mathrm{O}(2 \times 50 \mathrm{~mL})$, dried over $\mathrm{Na}_{2} \mathrm{SO}_{4}$, and finally the solvent was distilled off. Purification by column chromatography 
(eluent: $\left.\mathrm{CHCl}_{3}\right)$.Yield: $7.88 \mathrm{~g}(98.1 \%)$; colorless oil; ${ }^{1} \mathrm{H}-\mathrm{NMR}\left(200 \mathrm{MHz} ; \mathrm{CDCl}_{3} ; \mathrm{J} / \mathrm{Hz}\right): \delta=$ 7.2-7.3 (m, $2 \mathrm{H}, \mathrm{Ar}-\mathrm{H}), 6.71\left(\mathrm{~d}, 1 \mathrm{H},{ }^{3} J(\mathrm{H}, \mathrm{H})\right.$ 8.5, Ar-H), 5.95-6.07 (m, $\left.1 \mathrm{H}, \mathrm{CH}=\right), 5.38(\mathrm{~m}$, $\left.1 \mathrm{H}, \mathrm{CH}_{\mathrm{A}} \mathrm{H}_{\mathrm{B}}=\right), 5.28\left(\mathrm{~m}, 1 \mathrm{H}, \mathrm{CH}_{\mathrm{A}} \mathbf{H}_{\mathrm{B}}=\right), 4.52\left(\mathrm{~m}, 2 \mathrm{H}, \mathrm{OCH}_{2}\right), 2.70\left(\mathrm{t}, 2 \mathrm{H},{ }^{3} J(\mathrm{H}, \mathrm{H}) 7.4\right.$, Ar- $\left.\mathrm{CH}_{2}\right), 1.8-2.2\left(\mathrm{~m}, 4 \mathrm{H}, \mathrm{CH}_{2} \mathrm{CH}_{2} \mathrm{CF}_{2}\right)$.

\subsubsection{3-[4-Bromo-2-(1H,1H,2H,2H,3H,3H-perfluorodecyl)phenoxy]propane-1,2-diol}

1-Allyloxy-4-bromo-2-(1H,1H,2H,2H,3H,3H-perfluorodecyl)-benzene (7.88 g, $12.7 \mathrm{mmol})$, and $N$-methylmorpholine- $N$-oxide ( $3 \mathrm{~mL}, 60 \%$ solution in water) were dissolved in acetone $(50 \mathrm{~mL})$. Osmium tetroxide $(1 \mathrm{~mL}, 4 \mathrm{mM}$ solution in tert-butanol) was added, and the solution was stirred for $2 \mathrm{~h}$ at $25{ }^{\circ} \mathrm{C}$. Afterwards, saturated aqueous $\mathrm{Na}_{2} \mathrm{SO}_{3}$ solution $(5 \mathrm{~mL})$ was added and the mixture was stirred for $30 \mathrm{~min}$ at $25^{\circ} \mathrm{C}$. The mixture was filtered over a silica bed. The residue was carefully washed twice with acetone $(50 \mathrm{~mL})$, and the solvent was evaporated in vacuo. The residue was dissolved in EtOAc $(100 \mathrm{~mL})$. The solution was washed with $10 \%$ aqueous $\mathrm{H}_{2} \mathrm{SO}_{4}(30 \mathrm{ml})$, saturated $\mathrm{NaHCO}_{3}$ solution $(30 \mathrm{~mL})$ and $\mathrm{H}_{2} \mathrm{O}(30 \mathrm{~mL})$. The organic layer was dried over $\mathrm{Na}_{2} \mathrm{SO}_{4}$, and the solvent was evaporated in vacuo. Purification was done by crystallization from hexane. Yield: $5.6 \mathrm{~g}(67.1 \%)$; colorless solid; mp: $112{ }^{\circ} \mathrm{C} .{ }^{1} \mathrm{H}-\mathrm{NMR}\left(200 \mathrm{MHz} ; \mathrm{CDCl}_{3} ; \mathrm{J} / \mathrm{Hz}\right): \delta=7.2-7.3(\mathrm{~m}, 2 \mathrm{H}, \mathrm{Ar}-\mathrm{H}), 6.73(\mathrm{~d}, 1 \mathrm{H}$, ${ }^{3} J(\mathrm{H}, \mathrm{H})$ 8.6, Ar-H), 3.7-4.2 (m, $\left.5 \mathrm{H}, \mathrm{OCH}_{2}, \mathrm{OCH}\right), 2.66\left(\mathrm{t}, 2 \mathrm{H},{ }^{3} J(\mathrm{H}, \mathrm{H}) 7.6, \mathrm{Ar}^{-\mathrm{CH}_{2}}\right), 2.42$ (b s, $2 \mathrm{H}, \mathrm{OH}), 1.8-2.2$ (m, $4 \mathrm{H}, \mathrm{CH}_{2} \mathrm{CH}_{2} \mathrm{CF}_{2}$ ).

\subsubsection{4-[4-Bromo-2-(1H,1H,2H,2H,3H,3H-perfluorooctyl)phenoxymethyl]- 2,2-dimethyl-1,3-dioxolane}

A mixture of 3-[4-bromo-2- $(1 \mathrm{H}, 1 \mathrm{H}, 2 \mathrm{H}, 2 \mathrm{H}, 3 \mathrm{H}, 3 \mathrm{H}$-perfluorodecyl)phenoxy]propane-1,2-diol $(2.0 \mathrm{~g}, 3.04 \mathrm{mmol})$, PPTS $(200 \mathrm{mg})$ and 2,2-dimethoxypropane $(25 \mathrm{~mL})$ was stirred at $25^{\circ} \mathrm{C}$ for $24 \mathrm{~h}$ (TLC). The solvent was distilled off in vacuum at $25^{\circ} \mathrm{C}$, the residue was dissolved in $\mathrm{Et}_{2} \mathrm{O}(100 \mathrm{~mL})$ and washed with aqueous $\mathrm{NaHCO}_{3}(2 \times 25 \mathrm{~mL}), \mathrm{H}_{2} \mathrm{O}(2 \times 25 \mathrm{~mL})$, brine $(2 \times 25 \mathrm{~mL})$, and dried over $\mathrm{Na}_{2} \mathrm{SO}_{4}$. The solvent was distilled off at a rotatory evaporator and the product was purified by column chromatography (eluent $\mathrm{CHCl}_{3}$ ). Yield: $1.4 \mathrm{~g}(46.1$ \%); yellow waxy solid; ${ }^{1} \mathrm{H}-\mathrm{NMR}\left(200 \mathrm{MHz} ; \mathrm{CDCl}_{3} ; \mathrm{J} / \mathrm{Hz}\right): \delta=7.2-7.3(\mathrm{~m}, 2 \mathrm{H}, \mathrm{Ar}-\mathrm{H}) 6.71$ $\left(\mathrm{d}, 1 \mathrm{H},{ }^{3} J(\mathrm{H}, \mathrm{H})\right.$ 8.6, Ar-H), 4.4-4.5, 3.8-4.2 (2 m, $\left.5 \mathrm{H}, \mathrm{OCH}_{2}, \mathrm{OCH}\right), 2.65\left(\mathrm{t}, 2 \mathrm{H},{ }^{3} \mathrm{~J}(\mathrm{H}, \mathrm{H})\right.$ 7.5, $\left.\mathrm{Ar}-\mathrm{CH}_{2}\right), 1.8-2.3\left(\mathrm{~m}, 4 \mathrm{H}, \mathrm{CH}_{2} \mathrm{CH}_{2} \mathrm{CF}_{2}\right), 1.43,1.37\left(2 \mathrm{~s}, 6 \mathrm{H}, \mathrm{CH}_{3}\right)$.

\subsubsection{4-[4'-Benzyloxy-3-(1H,1H,2H,2H,3H,3H-perfluorodecyl)biphenyl-4-yloxymethyl]- 2,2-dimethyl-1,3-dioxolane}

A mixture of 4-[4-bromo-2- $(1 \mathrm{H}, 1 \mathrm{H}, 2 \mathrm{H}, 2 \mathrm{H}, 3 \mathrm{H}, 3 \mathrm{H}$-perfluorooctyl)phenoxymethyl]-2,2dimethyl-1,3-dioxolane (1.0 g, $1.4 \mathrm{mmol})$, 4-benzyloxybenzeneboronic acid $(0.65 \mathrm{~g}, 2.9$ mmol $), \operatorname{Pd}\left(\mathrm{PPh}_{3}\right)_{4}(0.04 \mathrm{~g})$, ethyleneglycol dimethylether $(10 \mathrm{~mL})$, and saturated $\mathrm{NaHCO}_{3}$ solution $(6 \mathrm{~mL})$ was refluxed for $6 \mathrm{~h}$ under an argon atmosphere. After staying over night at $\mathrm{RT}$, the precipitate was filtered and dissolved in $\mathrm{CHCl}_{3}(50 \mathrm{~mL})$. The solution was dried over $\mathrm{Na}_{2} \mathrm{SO}_{4}$, filtered through silica gel and the silica gel was washed thoroughly with $\mathrm{CHCl}_{3}(100$ $\mathrm{mL}$ ), the solvent was evaporated and the product was purified by crystallization from $\mathrm{CDCl}_{3} / \mathrm{MeOH}$ 1:4. Yield: $0.7 \mathrm{~g}$ (62.9\%); colorless solid mp: $100{ }^{\circ} \mathrm{C} ;{ }^{1} \mathrm{H}-\mathrm{NMR}(200 \mathrm{MHz}$; $\left.\mathrm{CDCl}_{3} ; J / \mathrm{Hz}\right): \delta=7.2-7.5(\mathrm{~m}, 9 \mathrm{H}, \mathrm{Ar}-\mathrm{H}), 7.01\left(\mathrm{~d}, 2 \mathrm{H},{ }^{3} J(\mathrm{H}, \mathrm{H}) 8.8, \mathrm{Ar}-\mathrm{H}\right), 6.88(\mathrm{~d}, 1 \mathrm{H}$, $\left.{ }^{3} J(\mathrm{H}, \mathrm{H}) 8.3, \mathrm{Ar}-\mathrm{H}\right), 5.09\left(\mathrm{~s}, 2 \mathrm{H}, \mathrm{Ar}-\mathrm{CH}_{2} \mathrm{O}\right), 4.4-4.6,3.85-4.2\left(\mathrm{~m}, 5 \mathrm{H}, \mathrm{OCH}_{2}, \mathrm{OCH}\right), 2.70(\mathrm{t}$, $\left.2 \mathrm{H},{ }^{3} \mathrm{~J}(\mathrm{H}, \mathrm{H}) 7.3, \mathrm{Ar}-\mathrm{CH}_{2}\right), 1.8-2.3\left(\mathrm{~m}, 4 \mathrm{H}, \mathrm{CH}_{2} \mathrm{CH}_{2} \mathrm{CF}_{2}\right), 1.45,1.39\left(2 \mathrm{~s}, 6 \mathrm{H}, \mathrm{CH}_{3}\right)$.

\subsubsection{3-[4'-Benzyloxy-3-(1H,1H,2H,2H,3H,3H-perfluorodecyl)biphenyl-4-yloxy)- propane-1,2-diol}

A mixture of 4-[4'-benzyloxy-3- $(1 \mathrm{H}, 1 \mathrm{H}, 2 \mathrm{H}, 2 \mathrm{H}, 3 \mathrm{H}, 3 \mathrm{H}-$ perfluorodecyl)biphenyl-4-yloxymethyl]-2,2-dimethyl-1,3-dioxolane $(700 \mathrm{mg}, 0.88 \mathrm{mmol})$ and $10 \% \mathrm{HCl}(1 \mathrm{~mL})$ in $\mathrm{EtOH}(50$ $\mathrm{mL}$ ) was refluxed for $3 \mathrm{~h}$. The solvent was evaporated and the residue was filtered and 
washed with saturated $\mathrm{NaHCO}_{3}(2 \times 50 \mathrm{~mL})$ and $\mathrm{H}_{2} \mathrm{O}(2 \times 50 \mathrm{~mL})$. Crystallization from EtOH gave the product as colorless crystals. Yield: $620 \mathrm{mg}(92.7 \%)$; mp: $122{ }^{\circ} \mathrm{C}$; ${ }^{1} \mathrm{H}-\mathrm{NMR}(200$ $\left.\mathrm{MHz} ; \mathrm{CDCl}_{3} ; J / \mathrm{Hz}\right): \delta=7.2-7.5(\mathrm{~m}, 9 \mathrm{H}, \mathrm{Ar}-\mathrm{H}), 7.01\left(\mathrm{~d}, 2 \mathrm{H},{ }^{3} J(\mathrm{H}, \mathrm{H}) 8.8, \mathrm{Ar}-\mathrm{H}\right), 6.90(\mathrm{~d}, 1$ $\mathrm{H},{ }^{3} \mathrm{~J}(\mathrm{H}, \mathrm{H})$ 8.3, Ar-H), 5.09 (s, $\left.2 \mathrm{H}, \mathrm{Ar}-\mathrm{CH}_{2} \mathrm{O}\right), 3.7-4.2\left(\mathrm{~m}, 5 \mathrm{H}, \mathrm{OCH}_{2}, \mathrm{OCH}\right), 2.74(\mathrm{t}, 2 \mathrm{H}$, $\left.{ }^{3} J(\mathrm{H}, \mathrm{H}) 7.3, \mathrm{Ar}-\mathrm{CH}_{2}\right), 1.8-2.3\left(\mathrm{~m}, 4 \mathrm{H}, \mathrm{CH}_{2}\right)$.

\subsubsection{3-[4'-Hydroxy-3-(1H,1H,2H,2H,3H,3H-perfluorodecyl)biphenyl-4-yloxy]propane- 1,2-diol 2/7}

3-[4'-Benzyloxy-3-(1H,1H,2H,2H,3H,3H-perfluorodecyl)biphenyl-4-yloxy)propane-1,2-diol $(620 \mathrm{~g}, 0.81 \mathrm{mmol})$, was dissolved in EtOAc $(50 \mathrm{~mL})$, palladium on carbon $(0.03 \mathrm{~g})$ was added, and the solution was shaken in a hydrogen atmosphere at ambient pressure at $25^{\circ} \mathrm{C}$ for $36 \mathrm{~h}$ (TLC). The solution was filtered over a silica bed. The residue was carefully washed twice with EtOAc $(50 \mathrm{~mL})$, and the solvent was evaporated in vacuum. The product was purified by crystallization from $\mathrm{CHCl}_{3} / \mathrm{MeOH}$ 20:3. Yield: $400 \mathrm{mg}(73.7 \%)$; transition temperatures $\left({ }^{\circ} \mathrm{C}\right)$ : $\mathrm{Cr} 116\left(\mathrm{Col}_{\mathrm{r}} / \mathrm{c} 2 \mathrm{~mm}\right.$ 102) $\mathrm{Col}_{\mathrm{r}} / \mathrm{c} 2 \mathrm{~mm} 131 \mathrm{Lam}_{\mathrm{Sm}} 132$ Iso; $\mathrm{C}_{25} \mathrm{H}_{21} \mathrm{O}_{4} \mathrm{~F}_{15}$. Anal. Calcd.: C, 44.79, H, 3.16; Found: C, 44.66, H, 3.04; ${ }^{1} \mathrm{H}-\mathrm{NMR}(400 \mathrm{MHz}$; DMSO-D ; $J / \mathrm{Hz}): \delta=9.39(\mathrm{~s}, 1 \mathrm{H}, \mathrm{Ar}-\mathrm{OH}), 7.3-7.5(\mathrm{~m}, 4 \mathrm{H}, \mathrm{Ar}-\mathrm{H}), 6.96\left(\mathrm{~d}, 1 \mathrm{H},{ }^{3} J(\mathrm{H}, \mathrm{H}) 8.8, \operatorname{Ar}-\mathrm{H}\right)$, $6.80\left(\mathrm{~d}, 2 \mathrm{H},{ }^{3} J(\mathrm{H}, \mathrm{H}) 8.6, \operatorname{Ar}-\mathrm{H}\right), 4.87(\mathrm{~d}, 1 \mathrm{H}, J(\mathrm{H}, \mathrm{H}) 5.0, \mathrm{CHOH}), 4.61\left(\mathrm{t}, 1 \mathrm{H},{ }^{3} J(\mathrm{H}, \mathrm{H})\right.$ 5.6, $\left.\mathrm{CH}_{2} \mathrm{OH}\right), 3.7-4.0\left(\mathrm{~m}, 3 \mathrm{H}, \mathrm{ArOCH} \mathbf{H}_{2} \mathrm{OCHOH}\right), 3.48\left(\mathrm{~m}, 2 \mathrm{H}, \mathrm{CH}_{2} \mathrm{OH}\right), 2.72\left(\mathrm{t}, 2 \mathrm{H},{ }^{3} J(\mathrm{H}\right.$, H) 7.4, $\left.\mathrm{ArCH}_{2}\right), 2.25\left(2 \mathrm{H}, \mathrm{CH}_{2} \mathrm{CF}_{2}\right), 1.84\left(\mathrm{~m}, 2 \mathrm{H}, \mathrm{CH}_{2} \mathrm{CH}_{2} \mathrm{CF}_{2}\right) ;{ }^{13} \mathrm{C}-\mathrm{NMR}(100 \mathrm{MHz}$; DMSO-D $\left._{6} ; J / \mathrm{Hz}\right): \delta=156.5,155.6,132.6,130.9,129.4,127.6,127.3(2 \mathrm{C}), 125.0,115.7(2 \mathrm{C})$, 112.1, 70.2, 69.8, 62.9, 29.9 (t), 29.1, 20.4; ${ }^{19} \mathrm{~F}-\mathrm{NMR}\left(188 \mathrm{MHz} ; \mathrm{DMSO}-\mathrm{D}_{6} ; J / \mathrm{Hz}\right): \delta=-80.8$ (t, $\left.3 \mathrm{~F}, \mathrm{CF}_{3}\right),-113.5\left(\mathrm{t}, 2 \mathrm{~F}, \mathrm{CH}_{2} \mathrm{CF}_{2}\right),-121.9\left(\mathrm{~m}, 2 \mathrm{~F}, \mathrm{CH}_{2} \mathrm{CF}_{2} \mathrm{CF}_{2}\right),-122.2$ (m, $2 \mathrm{~F}$, $\left.\mathrm{CH}_{2}\left(\mathrm{CF}_{2}\right)_{2} \mathrm{CF}_{2}\right)$. $-122.8\left(\mathrm{~m}, 2 \mathrm{~F}, \mathrm{CF}_{2}\left(\mathrm{CF}_{2}\right)_{2} \mathrm{CF}_{3}\right),-123.3\left(\mathrm{~m}, 2 \mathrm{~F}, \mathrm{CF}_{3} \mathrm{CF}_{2} \mathrm{CF}_{2}\right),-126.0(\mathrm{t}, 2 \mathrm{~F}$, $\mathrm{CF}_{3} \mathrm{CF}_{2}$ ).

\subsection{Analytical data of compound $2 / n$}

\subsubsection{3-[4'-Hydroxy-3-(1H,1H,2H,2H,3H,3H-perfluorohexyl)biphenyl-4-yloxy]propane- 1,2-diol 2/3}

Purification by crystallization from EE/hexane 2:6 Yield: $300 \mathrm{mg}(58.0 \%)$; transition temperatures $\left({ }^{\circ} \mathrm{C}\right)$ : $\mathrm{Cr} 98\left(\mathrm{Col}_{\mathrm{r}} / p 2 m m\right.$ 96) Iso; $\mathrm{C}_{21} \mathrm{H}_{21} \mathrm{O}_{4} \mathrm{~F}_{7}$. Anal. Calcd.: $\mathrm{C}, 53.62, \mathrm{H}, 4.50$; Found: C, 53.30, H, 4.99; ${ }^{1} \mathrm{H}-\mathrm{NMR}(400 \mathrm{MHz}$; DMSO-D $; J / \mathrm{Hz}): \delta=9.39(\mathrm{~s}, 1 \mathrm{H}, \mathrm{Ar}-\mathrm{OH})$, $7.41\left(\mathrm{~d}, 2 \mathrm{H},{ }^{3} J(\mathrm{H}, \mathrm{H}) \mathrm{8} .6, \mathrm{Ar}-\mathrm{H}\right), 7.35$ (m, $\left.2 \mathrm{H}, \mathrm{Ar}-\mathrm{H}\right), 6.96\left(\mathrm{~d},{ }^{3} J(\mathrm{H}, \mathrm{H}) 8.7,1 \mathrm{H}, \mathrm{Ar}-\mathrm{H}\right), 6.81$ $\left(\mathrm{d},{ }^{3} J(\mathrm{H}, \mathrm{H}) 8.6,1 \mathrm{H}, \mathrm{Ar}-\mathrm{H}\right), 4.88(\mathrm{~d}, J(\mathrm{H}, \mathrm{H}) 5.3,1 \mathrm{H}, \mathrm{CHOH}), 4.62\left(\mathrm{~m}, 1 \mathrm{H}, \mathrm{CH}_{2} \mathrm{OH}\right), 4.0-$ $3.8\left(\mathrm{~m}, 3 \mathrm{H}, \mathrm{ArOCH} \mathbf{H}_{2} \mathrm{OCHOH}\right), 3.48\left(\mathrm{~m}, 2 \mathrm{H}, \mathrm{CH}_{2} \mathrm{OH}\right), 2.70\left(\mathrm{t}, 2 \mathrm{H},{ }^{3} J(\mathrm{H}, \mathrm{H}) 7.5, \mathrm{Ar}_{-} \mathrm{CH}_{2}\right)$, $2.49\left(2 \mathrm{H}, \mathrm{CH}_{2} \mathrm{CF}_{2}\right), 1.84\left(\mathrm{~m}, 2 \mathrm{H}, \mathrm{CH}_{2} \mathrm{CH}_{2} \mathrm{CF}_{2}\right) ;{ }^{13} \mathrm{C}-\mathrm{NMR}(100 \mathrm{MHz}$; DMSO-D $; ~ J / H z): \delta=$ 156.4, 155.4, 132.4, 130.8, 129.2, 127.5, 127.1 (2C), 124.9, 115.5 (2C), 112.0, 70.0, 69.6, 62.7, $29.3(\mathrm{t}), 28.9,20.2 ;{ }^{19} \mathrm{~F}-\mathrm{NMR}\left(188 \mathrm{MHz} ; \mathrm{DMSO}^{-\mathrm{D}_{6}} ; \mathrm{J} / \mathrm{Hz}\right): \delta=-80.6\left(\mathrm{t}, 3 \mathrm{~F}, \mathrm{CF}_{3}\right)$, -114.4 (m, $\left.2 \mathrm{~F}, \mathrm{CH}_{2} \mathrm{CF}_{2}\right),-127.5\left(\mathrm{~s}, 2 \mathrm{~F}, \mathrm{CF}_{3} \mathrm{CF}_{2}\right)$.

\subsubsection{3-[4'-Hydroxy-3-(1H,1H,2H,2H,3H,3H-perfluoroheptyl)biphenyl-4-yloxy]propane- 1,2-diol 2/4}

Purification by crystallization from EE/hexane 2:6 Yield: $700 \mathrm{mg}(65.4 \%)$; transition temperatures $\left({ }^{\circ} \mathrm{C}\right)$ : $\mathrm{Cr} 87 \mathrm{Col}_{\mathrm{r}} / \mathrm{c} 2 \mathrm{~mm} 117$ Iso; $\mathrm{C}_{22} \mathrm{H}_{21} \mathrm{O}_{4} \mathrm{~F}_{9}$. Anal. Calcd.: $\mathrm{C}, 50.78, \mathrm{H}, 4.07$; Found: C, 50.91, H, 4.12; ${ }^{1} \mathrm{H}-\mathrm{NMR}(400 \mathrm{MHz}$; acetone-D 6 ; J/Hz): $\delta=8.34(\mathrm{~s}, 1 \mathrm{H}$, Ar-OH), 7.35-7.45 (m, $4 \mathrm{H}, \mathrm{Ar}-\mathrm{H}), 7.02\left(\mathrm{~d}, 1 \mathrm{H},{ }^{3} J(\mathrm{H}, \mathrm{H}) 8.3\right.$, Ar-H), $6.88\left(\mathrm{~d}, 2 \mathrm{H},{ }^{3} J(\mathrm{H}, \mathrm{H}) 8.7\right.$, Ar-H), 4.0-4.2, 3.6-3.8 (2 m, $\left.7 \mathrm{H}, \mathrm{OH}, \mathrm{OCH}, \mathrm{OCH}_{2}\right), 2.8\left(\mathrm{~m}\right.$, overlapped by solvent, $\left.2 \mathrm{H}, \mathrm{Ar}-\mathrm{CH}_{2}\right)$ 2.2-2.4 (m, $2 \mathrm{H}, \mathrm{CH}_{2} \mathrm{CF}_{2}$ ), 1.9-2.0 (m, $\left.2 \mathrm{H}, \mathrm{CH}_{2} \mathrm{CH}_{2} \mathrm{CF}_{2}\right) ;{ }^{13} \mathrm{C}-\mathrm{NMR}\left(100 \mathrm{MHz}\right.$; acetone-D ${ }_{6}$; $J / \mathrm{Hz}): \delta=157.3,156.7,134.2,132.9,130.4,128.8,128.3$ (2C), 126.0, $116.4(2 \mathrm{C}), 112.2$, 
71.5, 70.5, 64.2, 30.7 (t) 21.4 (one peak overlapped by the solvent); ${ }^{19} \mathrm{~F}-\mathrm{NMR}$ (188 MHz; DMSO-D $\left._{6} ; J / H z\right): \delta=-82.5\left(\mathrm{t}, 3 \mathrm{~F}, \mathrm{CF}_{3}\right),-115.1\left(\mathrm{t}, 2 \mathrm{~F}, \mathrm{CH}_{2} \mathrm{CF}_{2}\right),-125.3(\mathrm{~s}, 2 \mathrm{~F}$, $\left.\mathrm{CF}_{3} \mathrm{CF}_{2} \mathrm{CF}_{2}\right),-127.0\left(\mathrm{~m}, 2 \mathrm{~F}, \mathrm{CF}_{3} \mathrm{CF}_{2}\right)$.

\subsubsection{3-[4'-Hydroxy-3-(1H,1H,2H,2H,3H,3H-perfluorononyl)biphenyl-4-yloxy]- propane-1,2-diol 2/6}

Purification by crystallization from $\mathrm{CHCl}_{3} / \mathrm{MeOH}$ 20:3. Yield: $290 \mathrm{~g}$ (47.7 \%); transition temperatures $\left({ }^{\circ} \mathrm{C}\right)$ : $\mathrm{Cr} 99 \mathrm{Col}_{\mathrm{h}} 125$ Iso; $\mathrm{C}_{24} \mathrm{H}_{21} \mathrm{O}_{4} \mathrm{~F}_{13}$. Anal. Calcd.: C, 46.46, H, 3.41; Found: C, 46.30, H, 3.92; ${ }^{1} \mathrm{H}-\mathrm{NMR}(200 \mathrm{MHz}$; DMSO-D 6 ; J/Hz): $\delta=9.38(\mathrm{~s}, 1 \mathrm{H}, \mathrm{Ar}-\mathrm{OH}), 7.39$ (d, $2 \mathrm{H},{ }^{3} J(\mathrm{H}, \mathrm{H})$ 8.6, Ar-H), 7.3-7.4 (m, $\left.2 \mathrm{H}, \mathrm{Ar}-\mathrm{H}\right), 6.97\left(\mathrm{~d}, 1 \mathrm{H},{ }^{3} J(\mathrm{H}, \mathrm{H}) 9.2, \mathrm{Ar}-\mathrm{H}\right), 6.80(\mathrm{~d}$, ${ }^{3} J(\mathrm{H}, \mathrm{H})$ 8.6, $\left.2 \mathrm{H}, \mathrm{Ar}-\mathrm{H}\right), 4.8\left(\mathrm{~d}, 1 \mathrm{H},{ }^{3} J(\mathrm{H}, \mathrm{H}) 4.9, \mathrm{CHOH}\right), 4.60\left(\mathrm{t}, 2 \mathrm{H},{ }^{3} J(\mathrm{H}, \mathrm{H}) 5.7\right.$, $\left.\mathrm{CH}_{2} \mathrm{OH}\right), 3.7-4.1\left(\mathrm{~m}, 3 \mathrm{H}, \mathrm{ArOC} \mathbf{H}_{2} \mathrm{CHOH},\right), 3.48\left(\mathrm{t}, 2 \mathrm{H},{ }^{3} \mathrm{~J}(\mathrm{H}, \mathrm{H}) 5.4, \mathrm{CH}_{2} \mathrm{OH}\right) 2.72\left(\mathrm{t},{ }^{3} J(\mathrm{H}\right.$, H) 7.0, $\left.2 \mathrm{H}, \mathrm{Ar}-\mathrm{CH}_{2}\right), 2.26\left(\mathrm{~m}, 2 \mathrm{H}, \mathrm{CH}_{2} \mathrm{CF}_{2}\right), 1.2-1.8\left(\mathrm{~m}, 2 \mathrm{H}, \mathrm{CH}_{2} \mathrm{CH}_{2} \mathrm{CF}_{2}\right) ;{ }^{3} \mathrm{C}-\mathrm{NMR}(100$ MHz; DMSO-D $\left.\mathrm{D}_{6} ; \mathrm{J} / \mathrm{Hz}\right): \delta=152.5,151.5,128.5,126.8,125.7,123.5,123.2(2 \mathrm{C}), 120.9$, 111.5 (2C), 118.0, 65.9, 65.5, 58.6, 25.4 (t), 24.7, 16.0; ${ }^{19} \mathrm{~F}-\mathrm{NMR}(188 \mathrm{MHz}$; DMSO-D $J / \mathrm{Hz}): \delta=-77.1\left(\mathrm{t}, 3 \mathrm{~F}, \mathrm{CF}_{3}\right),-110.0\left(\mathrm{~s}, 2 \mathrm{~F}, \mathrm{CH}_{2} \mathrm{CF}_{2}\right),-118.6\left(\mathrm{~s}, 2 \mathrm{~F}, \mathrm{CH}_{2} \mathrm{CF}_{2} \mathrm{CF}_{2}\right),-119.5(\mathrm{~s}$, $\left.2 \mathrm{~F}, \mathrm{CF}_{3} \mathrm{CF}_{2} \mathrm{CF}_{2} \mathrm{CF}_{2}\right),-119.9$ (s, $\left.2 \mathrm{~F}, \mathrm{CF}_{2} \mathrm{CF}_{2} \mathrm{CF}_{3}\right)$, -122.6 (m, $\left.2 \mathrm{~F}, \mathrm{CF}_{3} \mathrm{CF}_{2}\right)$.

\subsubsection{3-[4'-Hydroxy-3-(1H,1H,2H,2H,3H,3H-perfluoroisodecyl)biphenyl-4-yloxy]- propane-1,2-diol 2/i7}

Purification by crystallization from EE/hexane 2:1. Yield: $600 \mathrm{mg}(74.6 \%)$; transition temperatures $\left({ }^{\circ} \mathrm{C}\right): \mathrm{Cr}<20 \mathrm{Col}_{\mathrm{r}} / \mathrm{c} 2 \mathrm{~mm} 131$ Iso; $\mathrm{C}_{25} \mathrm{H}_{21} \mathrm{O}_{4} \mathrm{~F}_{15}$. Anal. Calcd.: $\mathrm{C}, 44.79, \mathrm{H}, 3.16$; Found: C, 44.53, H, 3.51; ${ }^{1} \mathrm{H}-\mathrm{NMR}(200 \mathrm{MHz}$; DMSO-D $; J / \mathrm{Hz}): \delta=9.41(\mathrm{~s}, 1 \mathrm{H}, \mathrm{Ar}-\mathrm{OH})$, 7.4 (m, 4 H, Ar-H), 6.96 (d, 1 H, ${ }^{3} J(\mathrm{H}, \mathrm{H})$ 8.2, Ar-H), 6.79 (d, 2H, $\left.{ }^{3} J(\mathrm{H}, \mathrm{H}) 8.6, \mathrm{Ar}-\mathrm{H}\right), 4.89$ $(\mathrm{d}, J(\mathrm{H}, \mathrm{H}) 4.1,1 \mathrm{H}, \mathrm{CHOH}), 4.63\left(\mathrm{t}, 1 \mathrm{H},{ }^{3} J(\mathrm{H}, \mathrm{H})\right.$ 85.7, $\left.\mathrm{CH}_{2} \mathrm{OH}\right), 3.75-4.05(\mathrm{~m}, 3 \mathrm{H}$, ArOCH $\left.\mathbf{H}_{2} \mathrm{OCHOH}\right), 3.47\left(\mathrm{~m}, 2 \mathrm{H}, \mathrm{CH}_{2} \mathrm{OH}\right), 2.72\left(\mathrm{t}, 2 \mathrm{H},{ }^{3} \mathrm{~J}(\mathrm{H}, \mathrm{H}) 7.1, \mathrm{Ar}-\mathrm{CH}_{2}\right), 2.1-2.4(\mathrm{~m}, 2$ $\left.\mathrm{H}, \mathrm{CH}_{2} \mathrm{CF}_{2}\right), 1.86\left(\mathrm{~m}, 2 \mathrm{H}, \mathrm{CH}_{2} \mathrm{CH}_{2} \mathrm{CF}_{2}\right) ;{ }^{13} \mathrm{C}-\mathrm{NMR}(100 \mathrm{MHz}$; DMSO-D $; J / \mathrm{Hz}): \delta=156.5$, 155.5, 132.5, 130.9, 129.3, 127.6, 127.2 (2C), 124.9, $115.6(2 \mathrm{C}), 112.0,70.0,69.6,62.7,28.9$, 20.2; ${ }^{19} \mathrm{~F}-\mathrm{NMR}(188 \mathrm{MHz}$; DMSO-D $; \mathrm{J} / \mathrm{Hz}): \delta=-72.0\left(\mathrm{~m}, 6 \mathrm{~F}, 2 \mathrm{CF}_{3}\right),-113.5(\mathrm{t}, 2 \mathrm{~F}$, $\left.\mathrm{CH}_{2} \mathrm{CF}_{2}\right),-115.4\left(\mathrm{~s}, 2 \mathrm{~F}, \mathrm{CH}_{2} \mathrm{CF}_{2} \mathrm{CF}_{2}\right),-121.0\left(\mathrm{~s}, 2 \mathrm{~F}, \mathrm{CH}_{2}\left(\mathrm{CF}_{2}\right)_{2} \mathrm{CF}_{2}\right),-123.0(\mathrm{t}, 2 \mathrm{~F}$, $\left.\mathrm{CF}_{2} \mathrm{CFCF}_{3}\right),-185.8\left(\mathrm{~s}, 1 \mathrm{~F}, \mathrm{CF}_{3} \mathrm{CF}\right)$.

\subsubsection{3-[4'-Hydroxy-3-(1H,1H,2H,2H,3H,3H-perfluoroundecyl)biphenyl-4-yloxy]- propane-1,2-diol $2 / 8$}

Purification by crystallization from $\mathrm{CHCl}_{3} / \mathrm{MeOH}$ 20:3. Yield: $1.0 \mathrm{~g}$ (77.4 \%); transition temperatures $\left({ }^{\circ} \mathrm{C}\right)$ : $\mathrm{Cr} 119 \mathrm{Lam}_{\mathrm{Sm}} 139$ Iso; $\mathrm{C}_{26} \mathrm{H}_{21} \mathrm{O}_{4} \mathrm{~F}_{17}$. Anal. Calcd.: C, 43.35, H, 2.94; Found: C, 43.30, H, 3.26; ${ }^{1} \mathrm{H}-\mathrm{NMR}(400 \mathrm{MHz}$; DMSO-D $; J / \mathrm{Hz}): \delta=9.37(\mathrm{~s}, 1 \mathrm{H}, \mathrm{Ar}-\mathrm{OH})$, $7.38\left(\mathrm{~d}, 2 \mathrm{H},{ }^{3} J(\mathrm{H}, \mathrm{H})\right.$ 8.6, Ar-H), $7.35(\mathrm{~m}, 2 \mathrm{H}, \mathrm{Ar}-\mathrm{H}), 6.96\left(\mathrm{~d}, 1 \mathrm{H},{ }^{3} J(\mathrm{H}, \mathrm{H}) 8.8, \mathrm{Ar}-\mathrm{H}\right), 6.79$ (d, $2 \mathrm{H},{ }^{3} J(\mathrm{H}, \mathrm{H})$ 8.6, Ar-H), $4.85(\mathrm{~d}, 1 \mathrm{H}, J(\mathrm{H}, \mathrm{H}) 5.2, \mathrm{CHOH}), 4.59\left(\mathrm{t}, 1 \mathrm{H},{ }^{3} J(\mathrm{H}, \mathrm{H}) 5.7\right.$, $\left.\mathrm{CH}_{2} \mathbf{O H}\right), 3.75-4.05\left(\mathrm{~m}, 3 \mathrm{H}, \mathrm{ArOCH} \mathbf{H}_{2} \mathrm{OCHOH}\right), 3.5\left(\mathrm{~m}, 2 \mathrm{H}, \mathrm{CH}_{2} \mathrm{OH}\right), 2.70\left(\mathrm{t}, 2 \mathrm{H},{ }^{3} \mathrm{~J}(\mathrm{H}, \mathrm{H})\right.$ 7.5, Ar- $\left.\mathrm{CH}_{2}\right), 2.2-2.3\left(\mathrm{~m}, 2 \mathrm{H}, \mathrm{CH}_{2} \mathrm{CF}_{2}\right), 1.8-1.85\left(\mathrm{~m}, 2 \mathrm{H}, \mathrm{CH}_{2} \mathrm{CH}_{2} \mathrm{CF}_{2}\right) ;{ }^{13} \mathrm{C}-\mathrm{NMR}(100$ MHz; DMSO-D $; J / H z): \delta=156.4,155.5,132.5,130.8,129.2,127.5,127.1$ (2C), 124.9, 115.5 (2C), 112.0, 70.0, 69.6, 62.7, 29.3 (t), 28.9, 20.2; ${ }^{19} \mathrm{~F}-\mathrm{NMR}$ (188 MHz; DMSO-D $J / \mathrm{Hz}): \delta=-77.3\left(\mathrm{~s}, 3 \mathrm{~F}, \mathrm{CF}_{3}\right),-110.1\left(\mathrm{~s}, 2 \mathrm{~F}, \mathrm{CH}_{2} \mathrm{CF}_{2}\right),-118.7\left(\mathrm{~s}, 6 \mathrm{~F}, \mathrm{CH}_{2} \mathrm{CF}_{2}\left(\mathrm{CF}_{2}\right)_{3}\right),-119.5$ (s, $\left.2 \mathrm{~F}, \mathrm{CF}_{2}\left(\mathrm{CF}_{2}\right)_{2} \mathrm{CF}_{3}\right),-112.0$ (s, $\left.2 \mathrm{~F}, \mathrm{CF}_{2} \mathrm{CF}_{2} \mathrm{CF}_{3}\right),-122.8$ (s, $2 \mathrm{~F}, \mathrm{CF}_{3} \mathrm{CF}_{2}$ ).

\subsubsection{3-[4'-Hydroxy-3-(1H,1H,2H,2H,3H,3H-perfluorotridecyl)biphenyl-4-yloxy]- propane-1,2-diol 2/10}

Purification by crystallization from hexane/ EtOAc 2:3. Yield: $1.1 \mathrm{~g}(62.8 \%)$; transition temperatures $\left({ }^{\circ} \mathrm{C}\right)$ : $\mathrm{Cr} 135 \mathrm{Lam}_{\mathrm{Sm}} 152 \mathrm{Lam}_{\mathrm{N}} 153 \mathrm{Lam}_{\text {Iso }} 156$ Iso; $\mathrm{C}_{28} \mathrm{H}_{21} \mathrm{O}_{4} \mathrm{~F}_{21}$. Anal. Calcd.: 
C, 40.99, H, 2.58; Found: C, 40.83, H, 2.99; ${ }^{1} \mathrm{H}-\mathrm{NMR}$ (400 MHz; DMSO-D 6 ; J/Hz): $\delta=9.36$ (s, $1 \mathrm{H}, \mathrm{Ar}-\mathrm{OH}), 7.32\left(\mathrm{~d}, 2 \mathrm{H},{ }^{3} J(\mathrm{H}, \mathrm{H}) 8.6, \mathrm{Ar}-\mathrm{H}\right), 7.3(\mathrm{~m}, 2 \mathrm{H}, \mathrm{Ar}-\mathrm{H}), 6.90\left(\mathrm{~d}, 1 \mathrm{H},{ }^{3} J(\mathrm{H}, \mathrm{H})\right.$ 8.4, Ar-H), $6.76\left(\mathrm{~d}, 2 \mathrm{H},{ }^{3} J(\mathrm{H}, \mathrm{H})\right.$ 8.6, Ar-H), $4.84(\mathrm{br} \mathrm{s}, 1 \mathrm{H}, \mathrm{CHOH}), 4.58$ (br s, $1 \mathrm{H}$, $\left.\mathrm{CH}_{2} \mathrm{OH}\right), 3.7-4.0\left(\mathrm{~m}, 3 \mathrm{H}, \mathrm{ArOC} \mathbf{H}_{2} \mathrm{OCHOH}\right), 3.46\left(\mathrm{~m}, 2 \mathrm{H}, \mathrm{CH}_{2} \mathrm{OH}\right), 2.62\left(\mathrm{t}, 2 \mathrm{H},{ }^{3} \mathrm{~J}(\mathrm{H}, \mathrm{H})\right.$ 7.2, Ar- $\left.\mathrm{CH}_{2}\right), 2.0-2.2\left(\mathrm{~m}, 2 \mathrm{H}, \mathrm{CH}_{2} \mathrm{CF}_{2}\right), 1.75$ (m, $\left.2 \mathrm{H}, \mathrm{CH}_{2} \mathrm{CH}_{2} \mathrm{CF}_{2}\right) ;{ }^{13} \mathrm{C}-\mathrm{NMR}(100 \mathrm{MHz}$; DMSO-D $\left._{6}, J / \mathrm{Hz}\right): \delta=156.6,155.6,132.7,131.1,129.4,127.4,127.3(2 \mathrm{C}), 125.1,115.6(2 \mathrm{C})$, $112.1,70.0,69.5,62.7,29.6(\mathrm{t}), 28.9,20.0 ;{ }^{19} \mathrm{~F}-\mathrm{NMR}(188 \mathrm{MHz}$; DMSO-D 6 ; J/Hz): $\delta=-82.5$ (t, $\left.3 \mathrm{~F}, \mathrm{CF}_{3}\right),-114.4\left(\mathrm{~s}, 2 \mathrm{~F}, \mathrm{CH}_{2} \mathrm{CF}_{2}\right),-122.7$ (s, $\left.12 \mathrm{~F}, \mathrm{CH}_{2} \mathrm{CF}_{2}\left(\mathrm{CF}_{2}\right)_{6}\right),-123.9$ (s, $2 \mathrm{~F}$, $\left.\mathrm{CF}_{2} \mathrm{CF}_{2} \mathrm{CF}_{3}\right),-127.3\left(\mathrm{~s}, 2 \mathrm{~F}, \mathrm{CF}_{2} \mathrm{CF}_{3}\right)$.

\subsubsection{3-[4'-Hydroxy-3-(1H,1H,2H,2H,3H,3H-perfluoropentadecyl)biphenyl-4-yloxy]- propane-1,2-diol $2 / 12$}

Purification by preparative centrifugal thin layer chromatography (eluent: $\mathrm{CHCl}_{3} / \mathrm{CH}_{3} \mathrm{OH}$ 10:2), followed by crystallization from hexane/ EtOAc 2:3. Yield: $75 \mathrm{mg}$ (27.6\%); transition temperatures ( $\left({ }^{\circ} \mathrm{C}\right)$ : $\mathrm{Cr} 154\left(\mathrm{Lam}_{\mathrm{N}} 142\right) \mathrm{Lam}_{\text {Iso }} 188$ Iso; $\mathrm{C}_{30} \mathrm{H}_{21} \mathrm{O}_{4} \mathrm{~F}_{25} ;{ }^{1} \mathrm{H}-\mathrm{NMR}(400 \mathrm{MHz}$; DMSO-D $\left._{6} ; J / \mathrm{Hz}\right): \delta=9.32(\mathrm{~s}, 1 \mathrm{H}, \mathrm{Ar}-\mathrm{OH}), 7.15-7.3(\mathrm{~m}, 4 \mathrm{H}, \mathrm{Ar}-\mathrm{H}), 6.85\left(\mathrm{~d}, 1 \mathrm{H},{ }^{3} J(\mathrm{H}, \mathrm{H})\right.$ 8.6, Ar-H), 6.73 (d, $2 \mathrm{H},{ }^{3} J(\mathrm{H}, \mathrm{H})$ 8.4, Ar-H), 4.81 (s br, $\left.1 \mathrm{H}, \mathrm{CHOH}\right), 4.55$ (s br, $1 \mathrm{H}$, $\left.\mathrm{CH}_{2} \mathrm{OH}\right), 3.7-4.0\left(\mathrm{~m}, 3 \mathrm{H}, \mathrm{ArOCH} \mathbf{H}_{2} \mathrm{OCHOH}\right), 3.5\left(\mathrm{~m}, 2 \mathrm{H}, \mathrm{CH}_{2} \mathrm{OH}\right), 2.56\left(\mathrm{~m}, 2 \mathrm{H}, \mathrm{Ar}-\mathrm{CH}_{2}\right)$, $2.0\left(\mathrm{~m}, 2 \mathrm{H}, \mathrm{CH}_{2} \mathrm{CF}_{2}\right), 1.7\left(\mathrm{~m}, 2 \mathrm{H}, \mathrm{CH}_{2} \mathrm{CH}_{2} \mathrm{CF}_{2}\right) ;{ }^{13} \mathrm{C}-\mathrm{NMR}(100 \mathrm{MHz}$; DMSO-D $)$ : $\delta=$ 156.6, 155.5, 132.7, 131.0, 129.4, 127.2, 127.1 (2C), 125.0, 115.6 (2C), 112.0, 70.0, 69.4, 62.7, $29.6(\mathrm{t}), 28.9,20.0 ;{ }^{19} \mathrm{~F}-\mathrm{NMR}\left(188 \mathrm{MHz} ; \mathrm{DMSO}_{6} ; \mathrm{J} / \mathrm{Hz}\right): \delta=-83.2\left(\mathrm{t}, 3 \mathrm{~F}, \mathrm{CF}_{3}\right)$, -114.8 (s, $2 \mathrm{~F}, \mathrm{CH}_{2} \mathrm{CF}_{2}$ ), -123.0 (s, $\left.16 \mathrm{~F}, \mathrm{CH}_{2} \mathrm{CF}_{2}\left(\mathrm{CF}_{2}\right)_{8}\right),-124.2$ (s, $\left.2 \mathrm{~F}, \mathrm{CF}_{2} \mathrm{CF}_{2} \mathrm{CF}_{3}\right),-127.9$ $\left(\mathrm{s}, 2 \mathrm{~F}, \mathrm{CF}_{2} \mathrm{CF}_{3}\right)$. 